\title{
Article
}

\section{Digital Histology by Phase Imaging Specific Biomarkers for Human Tumoral Tissues Discrimination}

\author{
José Luis Ganoza-Quintana, Félix Fanjul-Vélez * and José Luis Arce-Diego *
}

check for updates

Citation: Ganoza-Quintana, J.L.; Fanjul-Vélez, F.; Arce-Diego, J.L. Digital Histology by Phase Imaging Specific Biomarkers for Human Tumoral Tissues Discrimination. Appl. Sci. 2021, 11, 6142. https://doi.org/ 10.3390/app11136142

Academic Editor: Gloria Bueno

Received: 14 May 2021

Accepted: 29 June 2021

Published: 1 July 2021

Publisher's Note: MDPI stays neutral with regard to jurisdictional claims in published maps and institutional affiliations.

Copyright: (c) 2021 by the authors. Licensee MDPI, Basel, Switzerland. This article is an open access article distributed under the terms and conditions of the Creative Commons Attribution (CC BY) license (https:// creativecommons.org/licenses/by/ $4.0 /)$.
Biomedical Engineering Group, TEISA Department, University of Cantabria, Av de los Castros 46, 39005 Santander, Spain; jose-luis.ganoza@alumnos.unican.es

* Correspondence: fanjulf@unican.es (F.F.-V.); arcedj@unican.es (J.L.A.-D.); Tel.: +34-942206730 (F.F.-V.); $+34-942201545$ (J.L.A.-D.)

Featured Application: Digital histology for tumoral tissues discrimination.

Abstract: Histology is the diagnosis gold standard. Conventional biopsy presents artifacts, delays, or human bias. Digital histology includes automation and improved diagnosis. It digitalizes microscopic images of histological samples and analyzes similar parameters. The present approach proposes the novel use of phase contrast in clinical digital histology to improve diagnosis. The use of label-free fresh tissue slices prevents processing artifacts and reduces processing time. Phase contrast parameters are implemented and calculated: the external scale, the fractal dimension, the anisotropy factor, the scattering coefficient, and the refractive index variance. Images of healthy and tumoral samples of liver, colon, and kidney are employed. A total of 252 images with $10 \times, 20 \times$, and $40 \times$ magnifications are measured. Discrimination significance between healthy and tumoral tissues is assessed statistically with ANOVA ( $p$-value $<0.005)$. The analysis is made for each tissue type and for different magnifications. It shows a dependence on tissue type and image magnification. The $p$-value of the most significant parameters is below $10^{-5}$. Liver and colon tissues present a great overlap in significant phase contrast parameters. The $10 \times$ fractal dimension is significant for all tissue types under analysis. These results are promising for the use of phase contrast in digital histology clinical praxis.

Keywords: digital histology; phase contrast imaging; biomarkers; biomedical optics; fractal analysis

\section{Introduction}

Histopathology is the gold standard for diagnosis. Current methods are based on direct microscopic observation of stained tissue slices by the pathologist [1]. Conventional biopsy is particularly indicated for tumoral tissue discrimination, even in early stages. The procedure presents several limitations, including artifacts generated by tissue processing, delays in the diagnosis caused by manual screening, or errors provoked by the pathologist bias [1]. Digital histology contributes to the automation of the procedure and to improved diagnosis by means of more objective parameters. Digital histology is implemented by digitalizing microscopic intensity images of conventional biopsy samples. Afterwards, advanced image analysis algorithms can be applied, including pattern recognition or color enhancement [2]. Three-dimensional (3D) reconstructions can be made from a series of consecutive 2D samples. Fluorescence biomarkers can be also detected and delimited on the sample image. Several biomarkers can be processed on the same image, for instance in a multiplexed image [2]. Digital histology allows a deeper analysis of biopsy samples, including numerical analysis, and it facilitates sharing the images among centers by telepathology [2]. However, it is based on conventional histological samples, so the artifacts of histological processing are still present, and sample processing time is high. Furthermore, usually, applied image analysis techniques analyze similar information as that seen by pathologists [1,2]. 
Label-free imaging could further improve digital histology analysis. It would prevent processing artifacts and reduce processing time. One of the problems is that biological tissues do not provide sufficient intrinsic contrast to distinguish the structures of interest, and staining is needed. Biological tissues are also intrinsically highly turbid, due to strong variations of refractive index. This fact complicates the observation of internal structures without physical sectioning. Optical scattering plays a significant role in light-tissue interactions. Light scattering techniques have been widely used for label-free diagnosis with different setups [3]. Some of the techniques include optical sectioning, such as Optical Coherence Tomography $[4,5]$. The problem with optical sectioning is the lack of images with very high resolution: below 1 micron. Microscopic observations allow practically diffraction-limited resolution. The use of several intrinsic contrast parameters has also been proposed, such as polarimetry [6,7], fluorescence [8,9], or spectroscopy [10-13]. However, the contrast of these techniques is usually limited and requires extrinsic biomarkers in the case of fluorescence.

In this work, we propose a novel approach that consists of exploiting the high variation of refractive index of biological tissues for diagnosis while maintaining very high resolution. We propose to employ label-free fresh tissue slices and phase contrast imaging for biological sample analysis. This approach allows first to eliminate artifacts that may appear in the fixation and staining stages of conventional biopsy procedures. Those procedures can be avoided due to the intrinsic refractive index variance that phase contrast exploits. It also reduces the sample processing time. Phase contrast imaging provides novel tissue characteristics that are particularly relevant in morphostructural changes, which are typical in tumoral tissues. This technique could complement or even serve as the main basis for disease diagnosis [14]. The present approach is based on Quantitative Phase Imaging (QPI) techniques [15]. The power spectral density of the optical beam is modeled by a Von Karman spectrum that depends on both the optical beam and biological tissue parameters [16]. Several parameters of the biological tissue are obtained by phase imaging processing, such as the external scale, the fractal dimension, the anisotropy factor, the scattering coefficient, or the refractive index variance [17-21]. The outer scale and the fractal dimension are obtained by modeling histological images using fractals. Refractive index variance depends on the gradient of the histological images. The anisotropy factor and scattering coefficient are related by the scattering-phase theorem to the intensity gradient of the phase contrast image. Samples of healthy and tumoral liver, colon, and kidney are extracted and analyzed. Phase images are obtained, and phase contrast parameters are implemented and calculated. A statistical analysis is made in order to evaluate the significance in the difference of the parameters for healthy and tumoral tissues as a function of tissue type. The present approach can be easily implemented in clinical praxis by using phase contrast microscopy and dedicated software on fresh samples.

\section{Materials and Methods}

This section describes the materials and methods employed in the work. It includes the description of the histological samples, the phase contrast microscope, and the phasesensitive biomarkers that will be used.

\subsection{Histological Samples}

Fresh samples of healthy and tumoral tissues from conventional biopsies made routinely on patients were obtained, following ethical guidelines of the biobank at the Marqués de Valdecilla University Hospital. Biological samples of liver, colon, and kidney were employed. Samples were frozen and sliced by a microtome, with a thickness of less than $6 \mu \mathrm{m}$. No fixing or staining procedures were applied to the samples, as these processes could alter tissue properties, particularly refractive index. Samples were collected on treated microscopy glass slides. Samples were maintained at $4{ }^{\circ} \mathrm{C}$ for three hours before measurement by phase contrast microscopy. 


\subsection{Phase Contrast Microscopy}

Phase contrast microscopy (PCM) presents advantages over conventional microscopy. It allows label-free, noninvasive imaging of live cells [15]. The image field is the superposition of fields originating at the specimen. For coherent illumination, the image field $U$ can be decomposed into its spatial average $U_{0}$ and fluctuating component, $U_{1}(x, y)$ :

$$
U(x, y)=U_{0}+\left[U(x, y)-U_{0}\right]=U_{0}+U_{1}(x, y) .
$$

Taking the Fourier transform of Equation (1):

$$
\widetilde{U}\left(k_{x}, k_{y}\right)=\delta(0,0)+\widetilde{U}_{1}\left(k_{x}, k_{y}\right) .
$$

The average field $U_{0}$ is the unscattered field, which is focused on axis by the objective, while $U_{1}$ corresponds to the scattered, high-frequency component. The decomposition in Equation (1) describes the image field as the interference between the scattered and unscattered components. The intensity image corresponds to an interferogram in the spatial domain,

$$
I(x, y)=\left|U_{0}\right|^{2}+\left|U_{1}(x, y)\right|^{2}+2\left|U_{0}\right|\left|U_{1}(x, y)\right| \cos [\Delta \phi(x, y)]
$$

where $\Delta \phi$ is the phase difference between the scattered and unscattered fields. Although in the case of optically thin specimens, this phase $\Delta \phi$ exhibits small variations, the intensity is very sensitive to $\Delta \phi$ changes around $\Delta \phi=\pi / 2$. Shifting the phase of the unscattered light by $\pi / 2$ provides an increased intensity contrast. This can be accomplished by a phase contrast filter with the transfer function $a \cdot e^{i \alpha}$. Applying the function over a phase object of unit amplitude, for small $\phi$, and choosing $\alpha= \pm \pi / 2$, we obtain [15]:

$$
I(x, y)=a^{2} \pm 2 a \phi(x, y) .
$$

According to this expression, the unscattered light produces a linear intensity with respect to $\phi$. The attenuation factor can be employed to further improve contrast.

A phase contrast microscopy setup was employed. Samples were placed on a motorized stage for automatic spatial sweeping. Phase contrast was implemented by an external phase ring combined with several phase contrast objectives with magnifications of $10 \times$, $20 \times$, and $40 \times($ Nikon $10 \times$ Plan Fluor 0.30NA Ph1 DLL, $20 \times$ SPlan Fluor ELWD 0.45 NA ph1 ADM, and $40 \times$ SPlan Fluor ELWD 0.60 NA ph2 ADM). Images were recorded with a CCD camera (Orca-R2, Hamamatsu) and stored in gray-scale 12-bit format.

\subsection{Phase Contrast Parameters}

The distribution of refractive index in a general turbulent medium requires a statistical description of the propagating beam. In this case, no temporal variation is expected, as we are dealing with turbid biological samples. Consequently, the analysis of phase contrast parameters must be based on the theory of random processes.

The most relevant statistical characteristics are the first two moments of the refractive index spatial distribution: the mean value

$$
n_{0}(\overrightarrow{\boldsymbol{r}})=\langle n(\overrightarrow{\boldsymbol{r}})\rangle_{M}
$$

and its covariance function

$$
B_{n}\left(\overrightarrow{r_{1}}, \overrightarrow{r_{2}}\right)=\left\langle\left[n\left(\overrightarrow{r_{1}}\right)-n_{0}\left(\overrightarrow{r_{1}}\right)\right]\left[n\left(\overrightarrow{r_{2}}\right)-n_{0}\left(\overrightarrow{r_{2}}\right)\right]\right\rangle_{M} .
$$

In a field propagating through a weakly scattering medium, the associated dispersion relation can be expressed as 


$$
\left\langle k^{2}\right\rangle=n_{0}^{2} \beta_{0}^{2}\left(1+\frac{\sigma_{n}^{2}}{n_{0}^{2}}\right)
$$

where $\left\langle k^{2}\right\rangle$ is the second-order moment of the wavevector, $\left\langle k^{2}\right\rangle=\left\langle k_{x}^{2}\right\rangle+\left\langle k_{y}^{2}\right\rangle+\left\langle k_{z}^{2}\right\rangle$, with angular brackets denoting ensemble averaging, $n_{0}$ is the average refractive index, $\beta_{0}$ is the wavenumber in vacuum, and $\beta_{0}=\omega / c$, and $\sigma_{n}^{2}$ is the spatial variance of the refractive index. The refractive index variance of a transparent sample can be calculated from the measured phase contrast image as [18]

$$
\sigma_{n}^{2}=\frac{1}{\beta_{0}^{2}}\left\langle|\nabla \phi|^{2}\right\rangle
$$

Equation (8) shows that the refractive index variance can be extracted from the mean gradient intensity of the phase contrast images. The scattering coefficient $\left(\mu_{s}\right)$ can be also estimated as a phase contrast parameter. It can be calculated using the scattering-phase theorem [17]

$$
\mu_{s}=\frac{\left\langle\Delta \varnothing^{2}(r)\right\rangle_{r}}{L}
$$

where $\left\langle\Delta \varnothing^{2}(r)\right\rangle$ denotes the spatial variance of the phase and $L$ is the thickness of the tissue sample.

The anisotropy factor can be calculated, as biological tissues are intrinsically highly scattering media. The estimation employs the scattering-phase theorem and can be implemented by

$$
g=1-\frac{1}{2 k_{0}^{2}} \frac{\left\langle|\nabla[\varnothing(r)]|^{2}\right\rangle_{r}}{\left\langle\Delta \varnothing^{2}(r)\right\rangle_{r}^{2}}
$$

where $k_{0}$ is the incident wave vector and $\nabla[\varnothing(r)]$ is the phase gradient.

Optical turbulence is usually explained by the presence of irregularities in the refractive index: the so-called "turbulent eddies" [16]. They are due to fluctuations in various physical properties, such as temperature, pressure, and inhomogeneous concentration of species. Different parts of the sample can be mixed by external or internal factors, such as cell growth or fluid transfer. This fact provokes energy transfer among eddies of different sizes. The largest possible size of an eddy is defined as the outer scale $L_{0}$ of turbulence. Larger eddies break down into smaller ones until their size reaches the lower limit and energy dissipates. In order to study these characteristics in histological samples, the spectrum of index inhomogeneities is fitted to the equation [22]

$$
\Phi(\kappa)=\frac{4 \pi \sigma_{n}^{2} L_{0}^{2}(m-1)}{\left(1+\kappa^{2} L_{0}^{2}\right)^{m}}
$$

which is a Von Karman spectrum, with the exception of the exponent $m$, that can assume values other than 4/3. $m$ is approximately equal to one-half of the measured slope in the range of power-law scaling. Its magnitude is related to the fractal dimension of a two-dimensional surface, $d_{f}=4-m$.

The spatial distribution of the main components of biological tissues has been shown to present self-similarity [23]. This facilitates the potential use of fractal analysis in these structures [24]. The goal is to identify spatial statistical regularities that could be described by power laws. The fractal dimension defines the stability characteristics of an image regarding scale transformations. The fractal dimension of an image can be estimated by several techniques: box-counting, correlation, sandbox, or even Fourier spectrum [25]. The box counting method consists of a grid with boxes of size $\varepsilon$ superimposed on an image. 
The number of boxes containing any part of the figure is recorded as $N(\varepsilon)$. The procedure is repeated for different sizes of $\varepsilon$, and the fractal dimension $d_{f}$ is calculated as

$$
d_{f}=-\frac{\log [N(\varepsilon)]}{\log (\varepsilon)} .
$$

$d_{f}$ is usually calculated from the negative value of the slope of the linear regression of $\log [N(\varepsilon)]$ on $\log (\varepsilon)$, following

$$
\log [N(\varepsilon)]=-d_{f} \log (\varepsilon) .
$$

\section{Results}

The previously described procedure is applied to histological images of kidney, colon, and liver. Sixteen samples of healthy and tumoral tissues of each type are employed. The next sections show the results of the image acquisition, calculation of parameters, and statistical analysis.

\subsection{Phase Contrast Images}

Each histological sample is imaged by the previously described phase contrast microscopy setup. Images are obtained with $10 \times(866 \times 660 \mu \mathrm{m}$; pixel size $0.64 \mu \mathrm{m}), 20 \times$ $(433 \times 330 \mu \mathrm{m}$; pixel size $0.3225 \mu \mathrm{m})$, and $40 \times(219 \times 167 \mu \mathrm{m}$; pixel size $0.1632 \mu \mathrm{m})$ magnifications. Special attention is given to pixel saturation and field of view. A total of 252 images were acquired. Figures 1-3 show images of healthy and tumoral liver, colon, and kidney, respectively, at $10 \times, 20 \times$, and $40 \times$.

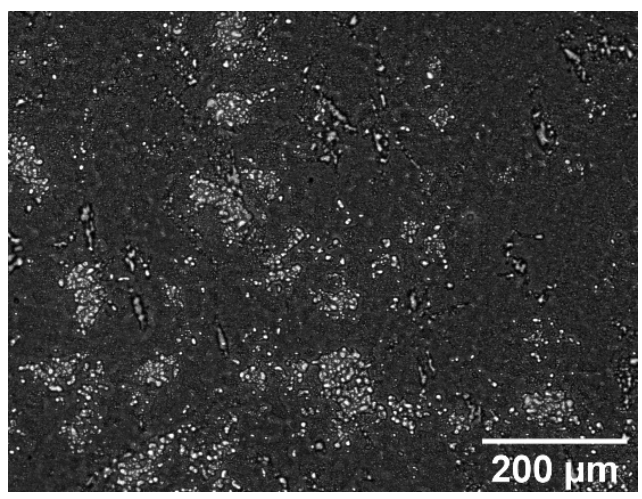

(a)

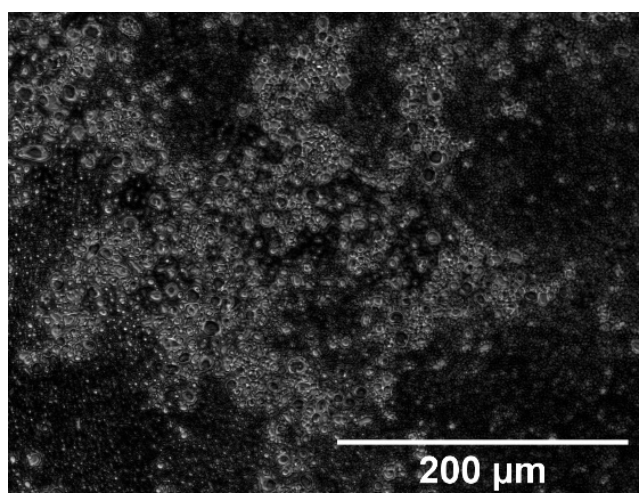

(c)

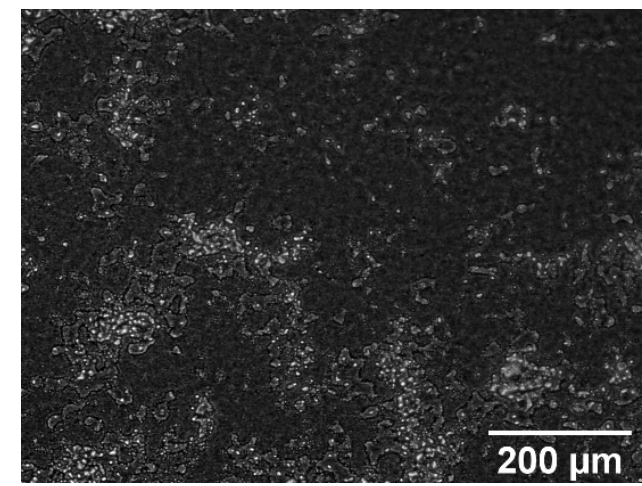

(b)

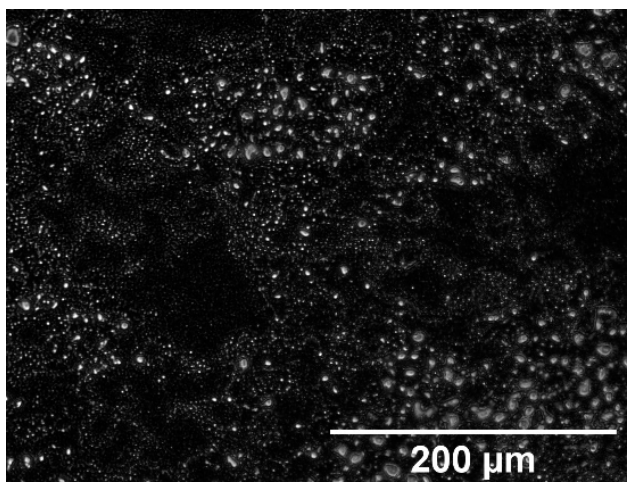

(d)

Figure 1. Cont. 


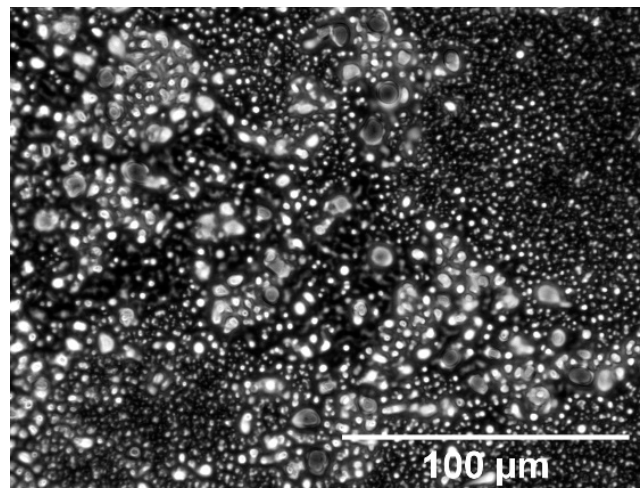

(e)

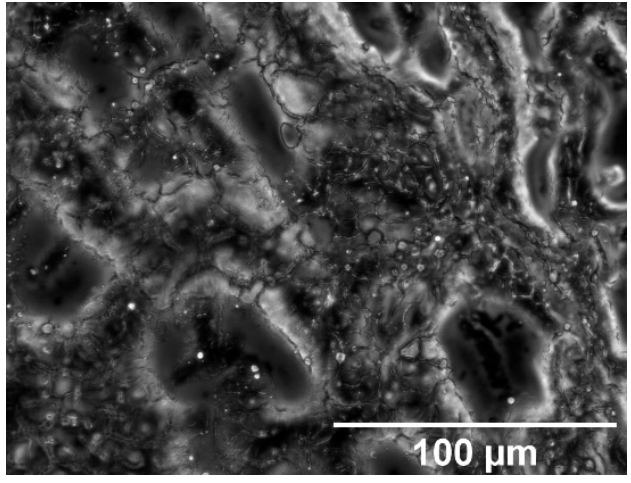

(f)

Figure 1. Histological images of healthy and tumoral liver tissue with different magnifications: (a) $10 \times$ healthy; (b) $10 \times$ tumoral; (c) $20 \times$ healthy; (d) $20 \times$ tumoral; (e) $40 \times$ healthy; (f) $40 \times$ tumoral. Hepatic cells and bile ducts can be appreciated. Tumoral images $(\mathbf{b}, \mathbf{d}, \mathbf{f})$ show a general tendency to structural disorder.

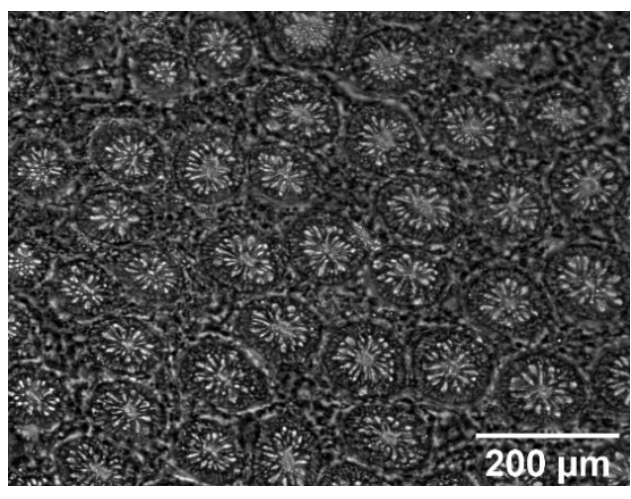

(a)

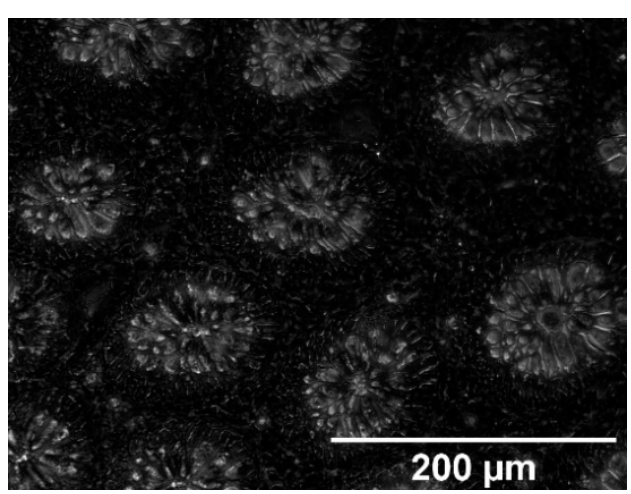

(c)

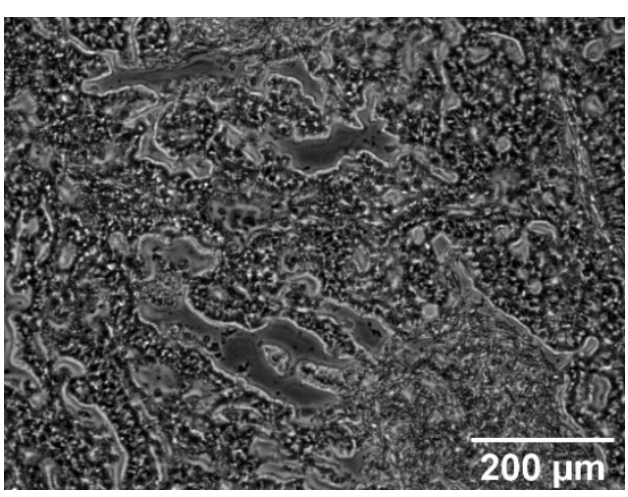

(b)

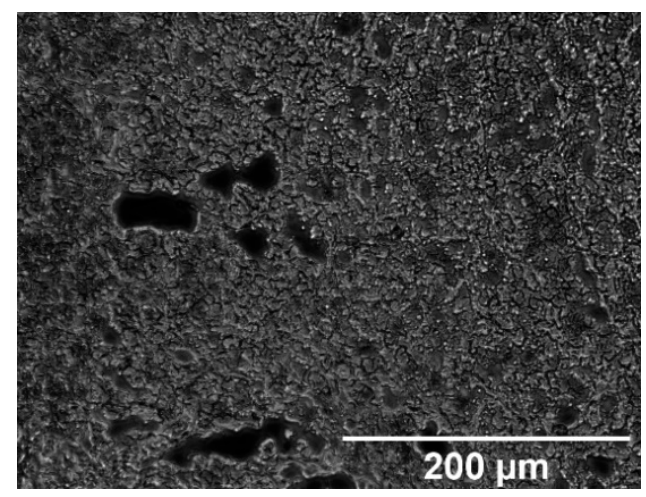

(d)

Figure 2. Cont. 


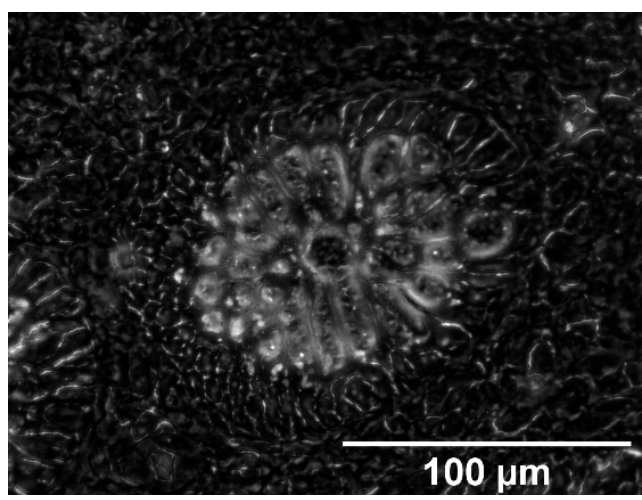

(e)

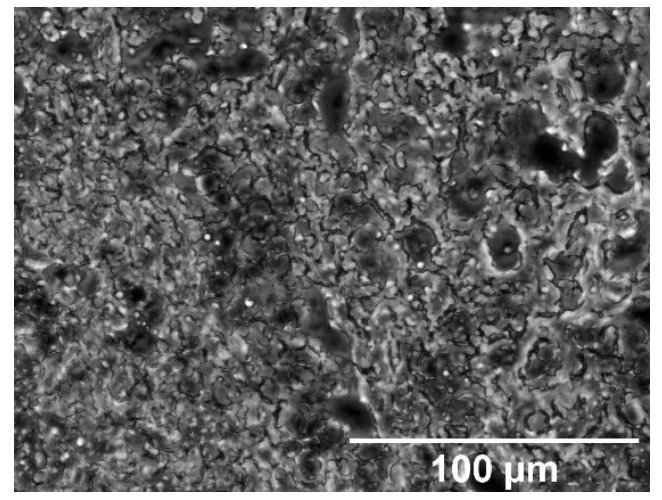

(f)

Figure 2. Histological images of healthy and tumoral colon tissue with different magnifications: (a) $10 \times$ healthy; (b) $10 \times$ tumoral; (c) $20 \times$ healthy; (d) $20 \times$ tumoral; (e) $40 \times$ healthy; (f) $40 \times$ tumoral. Healthy images $(\mathbf{a}, \mathbf{c}, \mathbf{e})$ show intestinal glands, while tumoral images $(\mathbf{b}, \mathbf{d}, \mathbf{f})$ show disordered structures as a consequence of the pathology.

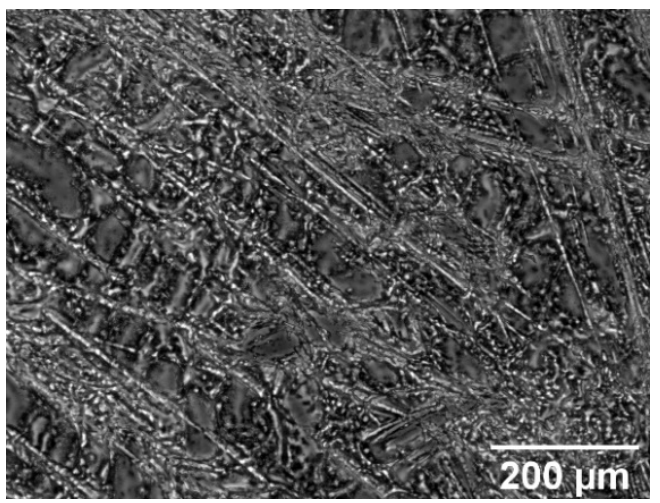

(a)

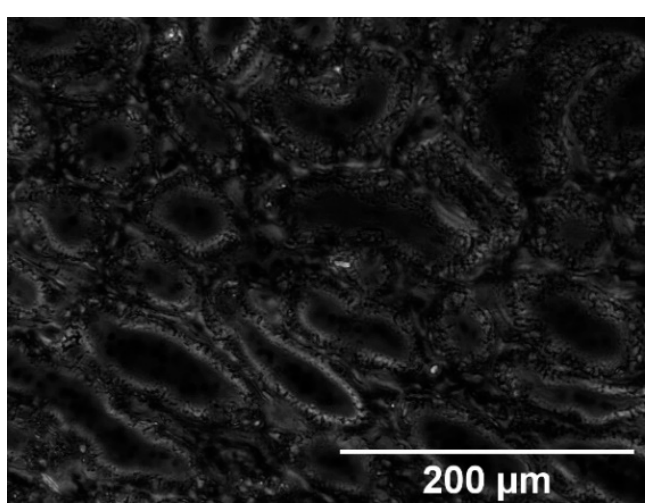

(c)

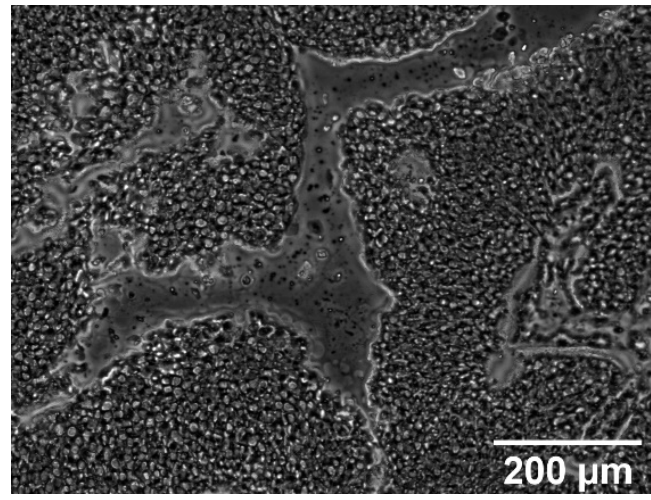

(b)

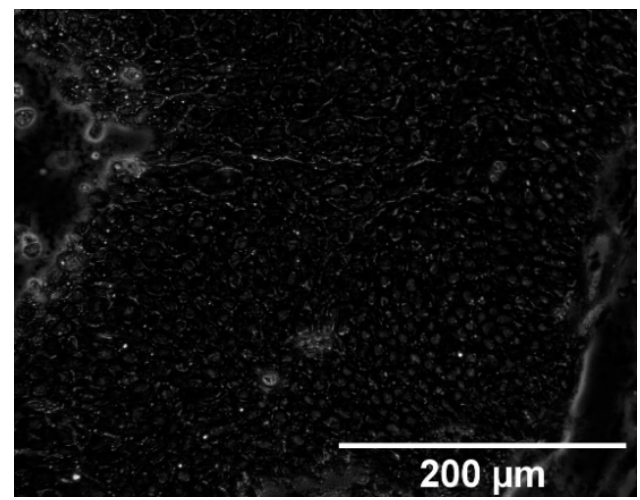

(d)

Figure 3. Cont. 


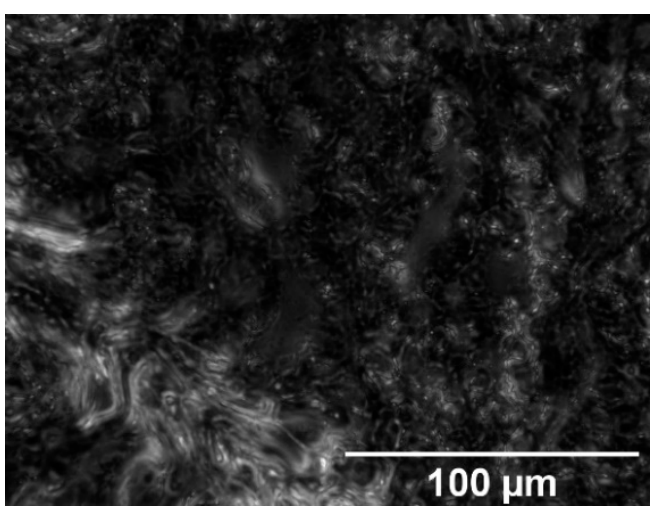

(e)

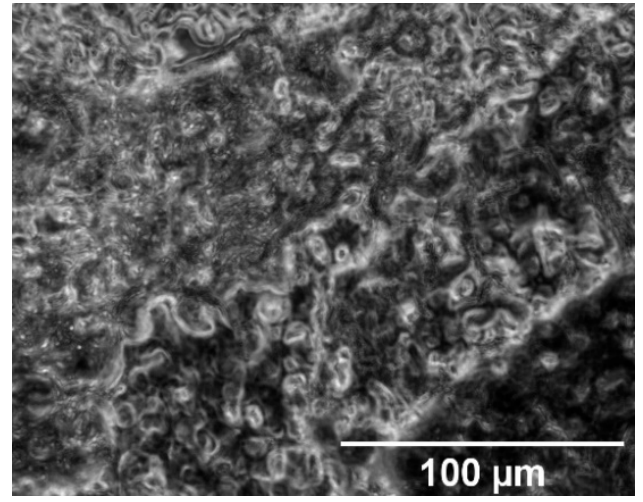

(f)

Figure 3. Histological images of healthy and tumoral kidney tissue with different magnifications: (a) $10 \times$ healthy; (b) $10 \times$ tumoral; (c) $20 \times$ healthy; (d) $20 \times$ tumoral; (e) $40 \times$ healthy; (f) $40 \times$ tumoral. Healthy images (a,c,e) show ordered structures, among others tubular structures, as corresponds to nephrons, glomeruli, and renal ducts. Tumoral images $(\mathbf{b}, \mathbf{d}, \mathbf{f})$ show a more homogeneous appearance, as a consequence of tumor growth.

Clear differences can be appreciated in these images, particularly regarding tissue structure, as expected. Phase information shows increased contrast in these fresh specimens. They would appear almost transparent under conventional microscopy. It is difficult to assess the pathological state from the direct observation of the images, particularly in Figure $1 c, d$, or in Figure $3 a, b$. The previously shown phase contrast parameters are calculated in the next section in order to solve this issue automatically.

\subsection{Phase Contrast Parameters Calculation}

The phase contrast parameters previously introduced and described in Section 2.3 are implemented in Matlab ${ }^{\circledR}$ and applied to the images. The results of the calculation are shown in Tables 1-3, depending on tissue type. The mean and standard deviation of each phase contrast parameter is obtained. The results are grouped by magnification factor, with the aim of healthy and tumoral tissues discrimination. For instance, in Table 1 for liver samples, refractive index variance is a better differentiation parameter for $10 \times$ magnification, compared to $20 \times$ and $40 \times$. Similarly, the scattering coefficient difference is more significant for $20 \times$ magnification in comparison with the results at $10 \times$ and $40 \times$.

Table 1. Results of phase contrast parameters for liver samples, at different magnifications, shown as mean \pm standard deviation.

\begin{tabular}{cccccc}
\hline Sample & \multirow{2}{*}{ RIV $^{\mathbf{1}}$} & $\begin{array}{c}\mathbf{S C}^{\mathbf{2}} \\
{\left[\mathbf{r a d}^{\mathbf{2}} \mathbf{m m}\right]}\end{array}$ & $\mathbf{A F}^{\mathbf{3}}$ & FD $^{\mathbf{4}}$ & OS $^{\mathbf{5}}[\boldsymbol{\mu m} \mathbf{m}$ \\
\hline $10 \times$ healthy & $0.0339 \pm 0.0045$ & $26.27 \pm 11.02$ & $0.97307 \pm 0.08658$ & $2.192 \pm 0.214$ & $81.72 \pm 11.91$ \\
$10 \times$ tumor & $0.0269 \pm 0.0060$ & $24.91 \pm 20.25$ & $0.99527 \pm 0.00531$ & $2.572 \pm 0.349$ & $88.27 \pm 10.73$ \\
$20 \times$ healthy & $0.0261 \pm 0.0098$ & $73.36 \pm 33.04$ & $0.99906 \pm 0.00017$ & $2.312 \pm 0.372$ & $40.24 \pm 4.71$ \\
$20 \times$ tumor & $0.0229 \pm 0.0048$ & $23.64 \pm 21.03$ & $0.99433 \pm 0.00713$ & $2.472 \pm 0.181$ & $40.63 \pm 7.72$ \\
$40 \times$ healthy & $0.0190 \pm 0.0071$ & $33.44 \pm 21.59$ & $0.99887 \pm 0.00039$ & $2.893 \pm 0.155$ & $25.84 \pm 3.77$ \\
$40 \times$ tumor & $0.0205 \pm 0.0045$ & $57.04 \pm 40.94$ & $0.99894 \pm 0.00070$ & $2.876 \pm 0.212$ & $22.87 \pm 5.52$ \\
\hline
\end{tabular}

${ }^{1}$ RIV $=$ Refractive Index Variance. ${ }^{2} \mathrm{SC}=$ Scattering Coefficient. ${ }^{3}$ AF $=$ Anisotropy Factor. ${ }^{4}$ FD $=$ Fractal Dimension. ${ }^{5}$ OS = Outer Scale. 
Table 2. Results of phase contrast parameters for colon samples, at different magnification, shown as mean \pm standard deviation.

\begin{tabular}{|c|c|c|c|c|c|}
\hline Sample & RIV $^{1}$ & $\begin{array}{c}\mathrm{SC}^{2} \\
{\left[\mathrm{rad}^{2} / \mathrm{mm}\right]}\end{array}$ & $\mathrm{AF}^{3}$ & FD $^{4}$ & $\mathrm{OS}^{5}[\mu \mathrm{m}]$ \\
\hline $10 \times$ healthy & $0.0299 \pm 0.0053$ & $22.55 \pm 9.67$ & $0.98495 \pm 0.06128$ & $2.358 \pm 0.242$ & $87.41 \pm 15.99$ \\
\hline $10 \times$ tumor & $0.0247 \pm 0.0053$ & $20.96 \pm 15.37$ & $0.99612 \pm 0.00395$ & $2.609 \pm 0.266$ & $84.35 \pm 11.26$ \\
\hline $20 \times$ healthy & $0.0228 \pm 0.0078$ & $56.21 \pm 30.09$ & $0.99877 \pm 0.00207$ & $2.476 \pm 0.324$ & $45.98 \pm 8.89$ \\
\hline $20 \times$ tumor & $0.0234 \pm 0.0043$ & $31.05 \pm 28.51$ & $0.99503 \pm 0.00594$ & $2.490 \pm 0.218$ & $42.67 \pm 10.53$ \\
\hline $40 \times$ healthy & $0.0182 \pm 0.0051$ & $47.01 \pm 25.30$ & $0.99911 \pm 0.00068$ & $2.998 \pm 0.162$ & $29.55 \pm 5.82$ \\
\hline $40 \times$ tumor & $0.0195 \pm 0.0037$ & $42.68 \pm 35.75$ & $0.99853 \pm 0.00124$ & $3.003 \pm 0.207$ & $25.28 \pm 5.53$ \\
\hline
\end{tabular}

Table 3. Results of phase contrast parameters for kidney samples, at different magnification, shown as mean \pm standard deviation.

\begin{tabular}{|c|c|c|c|c|c|}
\hline Sample & RIV $^{1}$ & $\begin{array}{c}\mathrm{SC}^{2} \\
{\left[\mathrm{rad}^{2} / \mathrm{mm}\right]}\end{array}$ & $\mathrm{AF}^{3}$ & FD $^{4}$ & $\mathrm{OS}^{5}[\mu \mathrm{m}]$ \\
\hline $10 \times$ healthy & $0.0244 \pm 0.0019$ & $28.35 \pm 5.13$ & $0.99821 \pm 0.00021$ & $2.722 \pm 0.104$ & $\begin{array}{c}118.89 \pm \\
48.99\end{array}$ \\
\hline $10 \times$ tumor & $0.0246 \pm 0.0022$ & $15.85 \pm 9.81$ & $0.99602 \pm 0.00141$ & $2.528 \pm 0.097$ & $97.87 \pm 19.89$ \\
\hline $20 \times$ healthy & $0.0200 \pm 0.0015$ & $51.38 \pm 25.40$ & $0.99808 \pm 0.00340$ & $2.764 \pm 0.079$ & $51.46 \pm 9.13$ \\
\hline $20 \times$ tumor & $0.0202 \pm 0.0042$ & $31.47 \pm 26.54$ & $0.99808 \pm 0.00152$ & $2.576 \pm 0.113$ & $45.84 \pm 10.41$ \\
\hline $40 \times$ healthy & $0.0156 \pm 0.0008$ & $30.65 \pm 31.55$ & $0.99617 \pm 0.00572$ & $3.256 \pm 0.089$ & $34.25 \pm 5.05$ \\
\hline $40 \times$ tumor & $0.0174 \pm 0.0019$ & $38.56 \pm 14.45$ & $0.99927 \pm 0.00022$ & $3.253 \pm 0.147$ & $29.92 \pm 6.47$ \\
\hline
\end{tabular}

Similar comparisons can be established for the other parameters. For instance, the anisotropy factor is quite similar between healthy and tumoral tissues for all types, even for different magnifications. Although there are promising parameters for healthy and tumoral tissue automatic classification [26,27], a deeper quantitative statistical analysis is needed. This analysis appears in the next section.

\subsection{Statistical Analysis}

The previous results are statistically analyzed. First, a boxplot analysis is made for each parameter as a function of tissue type and magnification. Selected results are shown in Figures 4 and 5. These figures show the median (red line), the distance between the first Q1 and third Q3 quartiles (blue box), and the distance between the maximum and minimum (dotted line). Figure 4 shows results for fractal dimension of liver tissue at $40 \times$, Figure $4 \mathrm{a}$, and refractive index variance of kidney tissue at $20 \times$, Figure $4 \mathrm{~b}$. In both cases, practically the entire range of variation of the healthy tissues is comprised in the variation range of the tumoral samples. As a consequence, measurements from both samples would be difficult to differentiate. Figure 5 represents the refractive index variance of colon tissue at $10 \times$, Figure $5 \mathrm{a}$, and scattering coefficient of liver tissue at $20 \times$, Figure $5 \mathrm{~b}$. In the former, there could be still a margin to potentially differentiate between the tissues, although there is a significant overlap. This process would be much easier in the latter case, Figure $5 b$, where there is practically no overlapping between both sample types. Consequently, the differentiation procedure would be easier. 


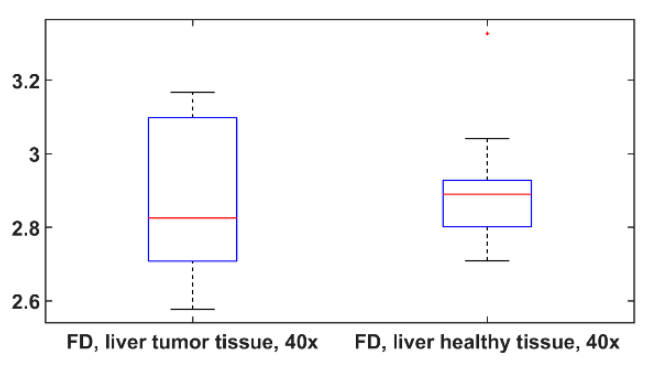

(a)

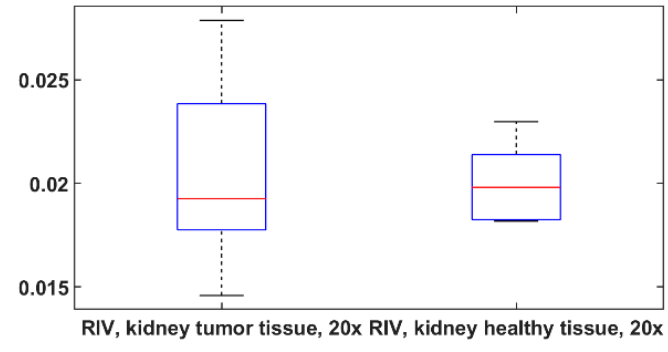

(b)

Figure 4. Boxplot corresponding to (a) fractal dimension (FD) of liver tissue at $40 \times$ and (b) refractive index variance (RIV) of kidney tissue at $20 \times$.

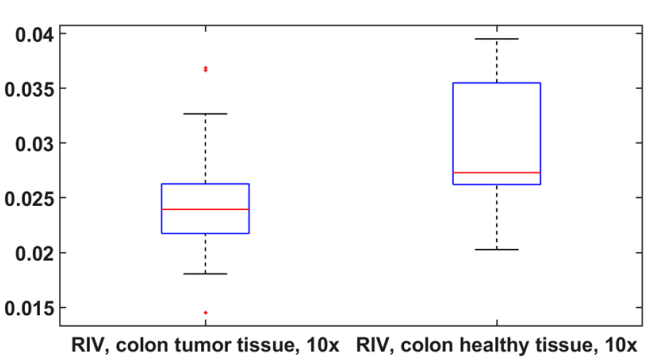

(a)

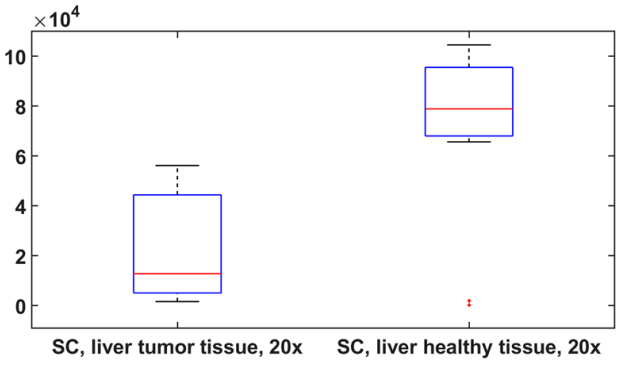

(b)

Figure 5. Boxplot corresponding to (a) refractive index variance (RIV) of colon tissue at $10 \times$ and (b) scattering coefficient (SC) of liver tissue at $20 \times$.

The previous boxplot analyses show the potentiality of some examples of phase contrast parameters for healthy and tumoral tissue discrimination. However, a deeper analysis is needed to study the significance of the parameters. An ANOVA statistical analysis is performed to analyze the significance of the potential average values of the parameters. The analysis is made for two different groups of healthy and tumoral tissues as a function of magnification. Tables 4-6 show the results of the ANOVA analysis for each tissue type. The results include the Snedecor F and p-values for each phase contrast parameter and magnification. The statistical test checks the hypothesis of equal means of the parameters. Therefore, there will be significant differences for large F values and correspondingly small $p$-values.

Table 4. Results of the ANOVA analysis for phase contrast parameters when comparing healthy and tumoral liver samples, for different magnifications.

\begin{tabular}{cccc}
\hline Parameter & Magnification & F & $p$-Value \\
\hline Anisotropy factor & $10 \times$ & 0.9166 & 0.3472 \\
& $20 \times$ & 6.1786 & 0.0197 \\
Scattering coefficient & $40 \times$ & 0.1183 & 0.7336 \\
& $10 \times$ & 0.0481 & 0.8282 \\
Fractal dimension & $20 \times$ & 22.5541 & 0.000065172 \\
& $40 \times$ & 3.6385 & 0.0676 \\
Outer scale & $10 \times$ & 11.9962 & 0.0019 \\
& $20 \times$ & 2.0859 & 0.1606 \\
& $40 \times$ & 0.0548 & 0.8168 \\
Refractive index variance & $10 \times$ & 2.2382 & 0.1472 \\
& $20 \times$ & 0.0262 & 0.8726 \\
& $40 \times$ & 2.4244 & 0.1331 \\
& $10 \times$ & 12.1175 & 0.0018 \\
& $20 \times$ & 1.2167 & 0.2801 \\
& $40 \times$ & 0.3927 & 0.5363 \\
\hline
\end{tabular}


Table 5. Results of the ANOVA analysis for phase contrast parameters when comparing healthy and tumoral colon samples, for different magnifications.

\begin{tabular}{cccc}
\hline Parameter & Magnification & $\mathbf{F}$ & $p$-Value \\
\hline Anisotropy factor & $10 \times$ & 0.9263 & 0.3401 \\
& $20 \times$ & 9.8824 & 0.0027 \\
Scattering coefficient & $40 \times$ & 4.6820 & 0.0349 \\
& $10 \times$ & 0.2152 & 0.6446 \\
Fractal dimension & $20 \times$ & 10.3137 & 0.0022 \\
& $40 \times$ & 0.2728 & 0.6036 \\
Outer scale & $10 \times$ & 13.5217 & 0.00054414 \\
& $20 \times$ & 0.0378 & 0.8466 \\
& $40 \times$ & 0.0107 & 0.9181 \\
& $10 \times$ & 0.6673 & 0.4176 \\
Refractive index variance & $20 \times$ & 1.5712 & 0.2155 \\
& $40 \times$ & 7.4722 & 0.0086 \\
& $10 \times$ & 13.0546 & 0.00066435 \\
& $20 \times$ & 0.1451 & 0.7047 \\
& $40 \times$ & 1.2440 & 0.2696 \\
\hline
\end{tabular}

Table 6. Results of the ANOVA analysis for phase contrast parameters when comparing healthy and tumoral kidney samples, for different magnifications.

\begin{tabular}{cccc}
\hline Parameter & Magnification & F & $p$-Value \\
\hline Anisotropy factor & $10 \times$ & 33.0444 & 0.0000047223 \\
& $20 \times$ & 0.00000087686 & 0.9993 \\
Scattering coefficient & $40 \times$ & 4.1214 & 0.0527 \\
& $10 \times$ & 17.8232 & 0.00026167 \\
Fractal dimension & $20 \times$ & 4.1114 & 0.0530 \\
& $40 \times$ & 0.7272 & 0.4016 \\
Outer scale & $10 \times$ & 25.8860 & 0.000026657 \\
& $20 \times$ & 25.5593 & 0.000029020 \\
& $40 \times$ & 0.0058 & 0.9399 \\
Refractive index variance & $10 \times$ & 2.2104 & 0.1491 \\
& $20 \times$ & 2.2326 & 0.1476 \\
& $40 \times$ & 3.8885 & 0.0593 \\
& $10 \times$ & 0.0780 & 0.7822 \\
& $20 \times$ & 0.0322 & 0.8590 \\
& $40 \times$ & 9.9905 & 0.0040 \\
\hline
\end{tabular}

\section{Discussion}

The results shown in the previous sections allow an estimation of the diagnostic potentiality of the novel phase contrast parameters proposed. The main difference between conventional or digital histology is based on the collection of phase information of the optical signal and the subsequent implementation of phase contrast parameters. This fact allows the use of fresh tissue slices that do not require fixation or staining, unlike conventional samples. This procedure decreases artifacts and processing time. Phase contrast images were obtained with a phase microscopy setup. Potentially relevant structures can be appreciated in Figures 1-3. They correspond to liver, colon, and kidney, either healthy or tumoral, and with different magnifications at $10 \times, 20 \times$, and $40 \times$. Each image was processed to calculate the proposed phase contrast parameters: anisotropy factor, scattering coefficient, fractal dimension, outer scale, and refractive index variance. These results are shown in Tables 1-3, for each tissue type, state, and magnification, and for selected cases in Figures 4 and 5. These results are a first approach to discrimination significance. For instance, in the case of liver at $10 \times$ (Table 1), refractive index variance and fractal dimension seem to have potentially usable differences. On the contrary, scattering coefficient, anisotropy of scattering, or outer scale do not seem to be useful, as they have values 
that overlap healthy and tumoral samples. The differences seem to be relevant for the scattering coefficient parameter in Table 1 for $20 \times$ compared with the rest of the phase contrast parameters. It is difficult to establish an a priori good differentiation parameter in the case of $40 \times$ magnification. These tendencies indicate that the validity of the parameters depends on the magnification factor. It is an expected result, considering that almost all the parameters are spatially sensitive. A similar analysis can be made in Table 2, which is devoted to colon tissues, or for Table 3, which contains kidney samples. A dependency on magnification and phase contrast parameters can be qualitatively stated. Figure 4 shows the cases of fractal dimension of liver tissue at 40x and refractive index variance of kidney tissue at $20 \times$. There is practically no area free from overlapping, so differentiation is expected to be complex. On the contrary, Figure 5 shows a refractive index variance of colon tissue at $10 \times$ and scattering coefficient of liver tissue at $20 \times$. In these examples, areas of no overlapping exist. This fact enormously facilitates the differentiation.

Although these analyses provide qualitative results, a deeper statistical analysis is made by using ANOVA, in order to better assess the potentiality of the phase contrast parameters. The results are shown in Tables 4-6, for the different samples of interest, liver, colon, and kidney. In these tables, a high Snedecor $\mathrm{F}$ value or, equivalently, a low $p$-value indicate that healthy and tumoral groups are statistically significant to the degree expressed by the $p$-value. The criterion of a $p$-value less than 0.005 is established to be considered statistically significant. With this criterion, for the liver case in Table 4, the scattering coefficient at $20 \times$, the fractal dimension at $10 \times$, and the refractive index variance at $10 \times$ would be significant. Particularly in the case of the scattering coefficient at $20 \times$, the significance would be greater. Interestingly, there are not significant parameters at $40 \times$ magnification for this tissue type, and two out of three belong to the $10 \times$ magnification. This fact stresses the relevance of the magnification factor of the images. This factor is intuitively related with the proposed phase contrast parameters, as they rely on spatially varying structures that are altered at different magnifications. In the case of colon samples, as shown in Table 5, the significant phase contrast parameters would be the anisotropy factor at 20x, the scattering coefficient at $20 \times$, the fractal dimension at $10 \times$, the outer scale at $40 \times$, and the refractive index variance at $10 \times$. Interestingly, these parameters are the same as those that affected liver tissue, but adding the anisotropy factor at $20 \times$ and the outer scale at $40 \times$. Nevertheless, the best significance results are for the fractal dimension at $10 \times$ and the refractive index variance at $10 \times$. These parameters do not coincide with the previous maximum significance parameter for liver tissue, which was the scattering coefficient at $20 \times$. This fact implies that there is a tissue type dependency in the selection of the optimum phase contrast parameter. According to the morphostructural differences of Figures 1-3, it is also an expected result. In the case of kidney, statistically significant parameters are the anisotropy factor at $10 \times$, scattering coefficient at $10 \times$, fractal dimensions at $10 \times$ and $20 \times$, and refractive index variance at $40 \times$. When compared with colon, there is a coincidence in the case of fractal dimension at $10 \times$, which was also present in liver. The other significant parameters are different, with changes in magnification with respect to colon, and lack of significance of the outer scale. Again, the rationale has to do with the structural difference of each tissue type and its relationship with the magnification factor.

These results show a promising applicability of phase contrast parameters in digital histology on fresh tissue samples. In particular, for the tissue samples under analysis, there is a coincidence of significance in the fractal dimension at $10 \times$ magnification. Other parameters, such as scattering coefficient at $20 \times$ or refractive index variance at $10 \times$, present significance in at least two tissue types. The results show that the clinical application of these parameters for a general cohort of biological samples would probably require the consideration of combined magnifications and parameters in a sophisticated classification algorithm. The fact that the samples are fresh could be on one side an advantage, as sample processing is eliminated and the procedure is faster. On the other side, it would be a difficult conservation that could only be done by congelation. Artifacts provoked in the fixation and staining phases are completely avoided. Novel diagnostic information of 
the samples is extracted, as phase data are discarded in conventional or digital histology. The potentiality of these novel parameters has been demonstrated. The implementation of the present approach in clinical praxis would require phase contrast microscopy and phase image analysis. The main procedure for fresh tissue samples would be the same as in conventional analysis, thus facilitating adoption.

\section{Conclusions}

In this work, phase contrast parameters have been proposed for the digital histology of phase contrast images of fresh biological tissue slices. Health and tumoral liver, colon, and kidney biological samples have been collected, sliced, and imaged by a phase contrast microscopy setup at $10 \times, 20 \times$, and $40 \times$ magnifications. Phase contrast parameters have been implemented and applied to the resulting images by image processing. These results have been statistically analyzed both qualitatively and quantitatively. The results of the analysis show a dependency of the significance with tissue type, magnification, and phase contrast parameter. However, the fractal dimension at $10 \times$ is significant in all the considered cases, and the scattering coefficient at $20 \times$ or refractive index variance at $10 \times$ are relevant in two-thirds of the samples.

This work has demonstrated the potentiality of phase contrast parameters for digital histology. This allows the future consideration of combined classification algorithms, as long as they provide an increase in diagnostic speed and accuracy over conventional or digital histology.

Author Contributions: Conceptualization, F.F.-V. and J.L.A.-D.; methodology, J.L.G.-Q., F.F.-V. and J.L.A.-D.; software, J.L.G.-Q. and F.F.-V.; validation, J.L.G.-Q., F.F.-V. and J.L.A.-D.; formal analysis, J.L.G.-Q., F.F.-V. and J.L.A.-D.; investigation, J.L.G.-Q., F.F.-V. and J.L.A.-D.; writing-original draft preparation, J.L.G.-Q. and F.F.-V.; writing-review and editing, F.F.-V. and J.L.A.-D.; supervision, F.F.-V. and J.L.A.-D. All authors have read and agreed to the published version of the manuscript.

Funding: This research was partially funded by the Spanish Ministry of Science, Research and Universities, cofunded by FEDER funds, "High-pressure driven plasmonic and luminescence properties of naked and core/shell metal-oxide nanocomposites", grant number PGC2018-101464-B-I00, and by San Cándido Foundation.

Institutional Review Board Statement: Not applicable.

Informed Consent Statement: Informed consent was obtained from all subjects involved in the study following ethical guidelines of the biobank.

Conflicts of Interest: The authors declare no conflict of interest.

\section{References}

1. Tortora, G.J.; Derrickson, B.H. Principles of Anatomy and Physiology, 15th ed.; Wiley: Hoboken, NJ, USA, 2018.

2. Yves, S.; Waelput, W. Digital Pathology; Springer: Berlin, Germany, 2014.

3. Tuchin, V.V. Tissue Optics: Light Scattering Methods and Instruments for Medical Diagnosis; Society of Photo-Optical Instrumentation Engineers (SPIE): Bellingham, WA, USA, 2015.

4. Brezinski, M.E.; Tearney, G.J;; Bouma, B.E.; Izatt, J.A.; Hee, M.R.; Swanson, E.A.; Southern, J.F.; Fujimoto, J.G. Optical coherence tomography for optical biopsy. Circulation 1996, 93, 1206-1213. [CrossRef]

5. Wang, R.K.; Tuchin, V.V. Advanced Biophotonics: Tissue Optical Sectioning; CRC Press: Boca Raton, FL, USA, 2014.

6. Pereda-Cubián, D.; Arce-Diego, J.L.; Rentmeesters, R. Characterization of depolarizing optical media by means of the entropy factor: Application to biological tissues. Appl. Opt. 2005, 44, 358-365. [CrossRef]

7. Ortega-Quijano, N.; Fanjul-Vélez, F.; Arce-Diego, J.L. Polarimetric study of birefringent turbid media with three-dimensional optic axis orientation. Biomed. Opt. Express 2014, 5, 287-292. [CrossRef] [PubMed]

8. Georgakoudi, I.; Jacobson, B.C.; van Dam, J.; Backman, V.; Wallace, M.B.; Müller, M.G.; Zhang, Q.; Badizadegan, K.; Sun, D.; Thomas, G.A.; et al. Fluorescence, reflectance, and light-scattering spectroscopy for evaluating dysplasia in patients with Barrett's esophagus. Gastroenterology 2001, 120, 1620-1629. [CrossRef] [PubMed]

9. Dhar, A.; Johnson, K.S.; Novelli, M.R.; Bown, S.G.; Bigio, I.J.; Lovat, L.B.; Bloom, S.L. Elastic scattering spectroscopy for the diagnosis of colonic lesions: Initial results of a novel optical biopsy technique. Gastrointest. Endosc. 2006, 63, 257-261. [CrossRef] [PubMed] 
10. Salas-García, I.; Fanjul-Vélez, F.; Arce-Diego, J.L. Influence of the human skin tumor type in photodynamic therapy analysed by a predictive model. Int. J. Photoenergy 2012, 2012, e759205. [CrossRef]

11. Pifferi, A.; Swartling, J.; Chikoidze, E.; Torricelli, A.; Taroni, P.; Bassi, A.L.; Andersson-Engels, S.; Cubeddu, R. Spectroscopic timeresolved diffuse reflectance and transmittance measurements of the female breast at different interfiber distances. J. Biomed. Opt. 2004, 9, 1143-1151. [CrossRef]

12. Amelink, A.; Robinson, D.J.; Sterenborg, H.J.C.M. Confidence intervals on fit parameters derived from optical reflectance spectroscopy measurements. J. Biomed. Opt. 2008, 13, 054044. [CrossRef]

13. Uttam, S.; Pham, H.V.; LaFace, J.; Leibowitz, B.; Yu, J.; Brand, R.E.; Hartman, D.J.; Liu, Y. Early prediction of cancer progression by depth-resolved nanoscale mapping of nuclear architecture from unstained tissue specimens. Cancer Res. 2015, 75, 4718-4727. [CrossRef]

14. Kumar, G.; Schmitt, J.M. Micro-optical properties of tissue. In Advances in Laser and Light Spectroscopy to Diagnose Cancer and Other Diseases III: Optical Biopsy. Int. Soc. Opt. Photonics 1996, 2679, 106-116.

15. Popescu, G. Quantitative Phase Imaging of Cells and Tissues; McGraw-Hill Education: New York, NY, USA, 2011.

16. Chen, X.; Li, J.; Korotkova, O. Light scintillation in soft biological tissues. Waves Random Complex Media 2020, $30,481-489$. [CrossRef]

17. Wang, Z.; Ding, H.; Popescu, G. Scattering-phase theorem. Opt. Lett. 2011, 36, 1215-1217. [CrossRef]

18. Shan, M.; Kandel, M.E.; Popescu, G. Refractive index variance of cells and tissues measured by quantitative phase imaging. Opt. Express 2017, 25, 1573-1581. [CrossRef]

19. Angelsky, O.V.; Maksimyak, P.P. Optical correlation diagnostics of random fields and objects. Opt. Eng. 1995, 34, 973-981. [CrossRef]

20. Vaezy, S.; Clark, J.I. Characterization of the cellular microstructure of ocular lens using 2D power law analysis. Ann. Biomed. Eng. 1995, 23, 482-490. [CrossRef]

21. Zwiggelaar, R.; Bull, C.R. Optical determination of fractal dimensions using Fourier transforms. Opt. Eng. 1995, 34, 1325-1332. [CrossRef]

22. Weiss, G. Wave Propagation in a Turbulent Medium. Tatarski, V.I., Ed.; Silverman, R.A., Translator; McGraw-Hill, New York, 1961. 285 Pp. Illus. \$9.75. Science 1961, 134, 324-325. [CrossRef]

23. Sheppard, C.J.R. Scattering by fractal surfaces with an outer scale. Opt. Commun. 1996, 122, 178-188. [CrossRef]

24. Sheppard, C.J.R. Fractal model of light scattering in biological tissue and cells. Opt. Lett. 2007, 32, 142-144. [CrossRef]

25. Baish, J.W.; Jain, R.K. Fractals and cancer. Cancer Res. 2000, 60, 3683-3688. [PubMed]

26. Fanjul-Vélez, F.; Arévalo-Díaz, L.; Arce-Diego, J.L. Intra-class variability in diffuse reflectance spectroscopy: Application to porcine adipose tissue. Biomed. Opt. Express 2018, 9, 2297-2303. [CrossRef] [PubMed]

27. Fanjul-Vélez, F.; Pampín-Suárez, S.; Arce-Diego, J.L. Application of classification algorithms to diffuse reflectance spectroscopy measurements for ex vivo characterization of biological tissues. Entropy 2020, 22, 736. [CrossRef] [PubMed] 